\title{
The molecular architecture of the TNF superfamily
}

\author{
J ean-Luc Bodmer, Pascal Schneider and J ürg Tschopp
}

\begin{abstract}
Ligands of the TNF (tumour necrosis factor) superfamily have pivotal roles in the organization and function of the immune system, and are implicated in the aetiology of several acquired and genetic diseases. TNF ligands sharea common structural motif, the TNF homology domain (THD), which binds to cysteine-rich domains (CRDs) of TNF receptors. CRDs are composed of structural modules, whose variation in number and type confers heterogeneity upon the family. Protein folds reminiscent of the THD and CRD are also found in other protein families, raising the possibility that the mode of interaction between TNF and TNF receptors might be conserved in other contexts.
\end{abstract}

Metazoan organisms consist of an intricateand ordered society of individual cells that must communicate to maintain and regulate their functions. This is achieved through a complex and highly regulated network of hormones, chemical mediators, chemokines and other cytokines, acting as ligands for intra- or extracellular receptors. Ligands and receptors of the tumour necrosis factor (TNF ) superfamilies are examples of signal transducers whose integrated actions impinge principally on the development, homeostasis and adaptativeresponses of the immune system. Despite their varied and plei otropic actions, members of the TNF ligand and receptor (TNFR) families have remarkably similar structures, and their mode of interaction is conserved. The aim of this review is to provide an overview of the molecular architecture and the modular organization of the TNF and TNFR gene superfamilies.

\section{The TNF family}

TheTNF ligand family comprises 18 genes encoding 19 typell (i.e. intracellular $\mathrm{N}$ terminus and extracellular C terminus) transmembrane proteins characterized by a conserved C-terminal domain coined the TNF homology domain' (THD) (Fig. 1). This trimeric domain is responsible for receptor binding and its sequence identity between family members is 20-30\%. Although most ligands are synthesized as membrane-bound proteins, soluble forms can be generated by limited proteolysis (Fig. 1). Distinct proteases are involved in this process,

J ürg Tschopp* Pascal Schneider J ean-Luc Bodmer Institute of Biochemistry, University of Lausanne Chemin des Boveresses 155, 1066 Epalinges Switzerland.

*e-mail:J urg.Tschopp@ ib.unil.ch depending on the ligand: metalloproteases of the ADAM (a disintegrin and metalloproteinase domain) family act on TNF and RANKL ligands [1,2], matrilysin acts on F as ligand (FasL) [3], and members of thesubtilisin-like furin family act on BAFF, EDA, TWEAK and APRIL-members of the TNF family [4,5]. Solubilization is absolutely required for the physiological function of some ligands: mutation in the furin recognition sequence of EDA is a frequent cause of the genetic disorder $X$-linked hypohidrotic ectodermal dysplasia (XLHED) $[4,6]$. By contrast, theshedding of some ligands inhibits their function. For instance, the cytotoxic activity of FasL is dramatically downregulated upon cleavage [7]. The $\mathrm{N}$ terminus of lymphotoxin $\alpha(\mathrm{LT} \alpha)$ resembles a signal peptide, making its conversion to a soluble form extremely efficient. Consequently, LT $\alpha$ is never found at the cell surface except when it is associated with membranebound LT $\beta$ as LT $\alpha_{1} \beta_{2}$ heterocomplexes [8] (Fig. 1). Processing of TNF-related apoptosis-inducing ligand (TRAIL) by a cysteine protease has been proposed [9], but the resulting sol uble form seems to betoo small to retain a functional THD.

Ligands of the TNF family control and orchestrate the immune and inflammatory responses at several levels (recently reviewed in Ref. [10]). During devel opment, TNF ligands such as TNF, LT $\alpha$, LT $\beta$ and RANKL providecrucial signals for the morphogenesis of secondary lymphoid organs [10,11]. In addition, the grooming and proper activation of immune precursor cells to fully competent effectors is dependent on several other TNF family members such as BAFF and CD40L for B lymphocytes [12-14]; 4-1BBL, OX40L and CD27L for T Iymphocytes [15]; and CD40L and RANKL for dendritic cells $[16,17]$. Pro-apoptotic members of the family (e.g. TNF, FasL and TRAIL ) contributeto the function of cytotoxic effector cells and participatein thehomeostasis of the lymphoid compartment by evoking activationinduced cell death in immune effector cells that have fulfilled their function [18]. Recent evidenceindicates that other TNF family ligands regulatethe development and differentiation of epithelial structures (theEDA ligand), endothelial cells (VEGI and TWEAK) and boneresorbing osteoclasts (RANKL and TNF) [10].

TNF family ligands and receptors areassociated with several diseaseconditions that result from acquired processes or genetic defects. Acquired acute or chronic inflammatory conditions such as septic shock or rheumatoid arthritis result from excessive or inappropriate TNF expression [19]. Mutations in TNF ligands and/or receptors have been described in five hereditary diseases: hyper I gM syndrome (HIM, CD40L), typel autoimmunelymphoproliferative syndrome(ALPS, Fas/F asL), TNF-R1-associated periodic fever syndrome (TRAPS, TNF-R1), 


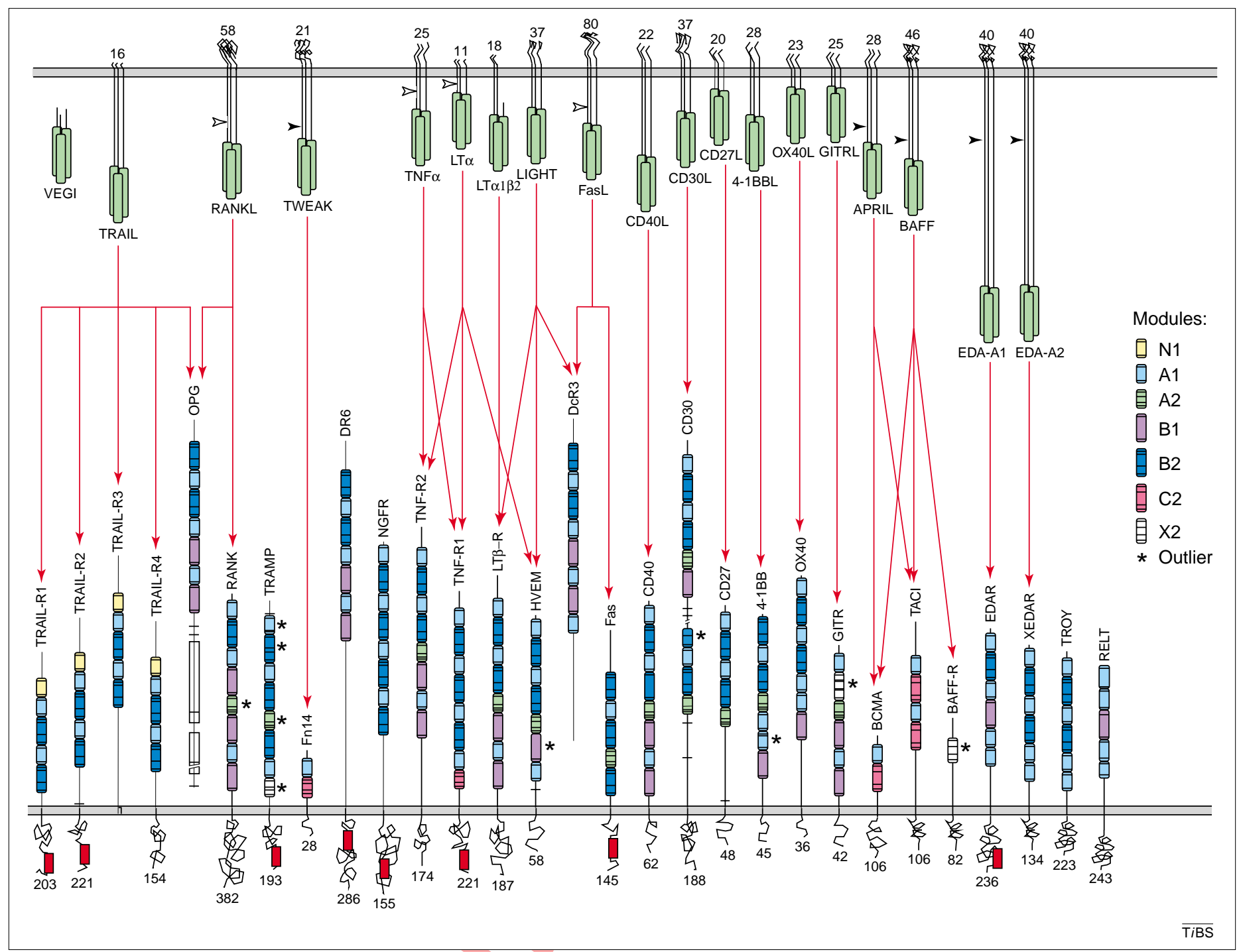

Fig. 1. Interactions between ligands and receptors of the human tumour necrosis factor (TNF) family: TNF ligands (top) and TNF receptors (bottom). The TNF ligands are represented as type II homo- or hetero-trimeric transmembrane proteins (with the exception of VEGI, which lacks a predicted transmembrane domain and is therefore drawn as a soluble ligand). TNF homology domains (THDs) are shown as green boxes. Filled black arrowheads indicate processing by furin family members, and open black arrowheads by other types of proteases. The TNF receptors are typically type I or type III transmembrane proteins, but also occur as glycolipid-anchored or soluble proteins. N1, A1, A2, B1, $\mathrm{B} 2, \mathrm{C} 2$ and $\mathrm{X} 2$ modules are colour-coded as shown in the insert. The positions of individual cysteines are indicated by horizontal bars, and stars show modules whose cysteine pattern does not conform entirely to that of cannonical A, B, Cand N modules. The length of intracellular domains is indicated for each ligand and each receptor, and the intracellular homology domain, known as the 'death domain', is indicated as red boxes. Red arrows show documented interactions. An interaction between TWEAK and TRAM P has been reported [53] but has not yet been confirmed. Some of the ligands and receptors have several commonly used names: FasL/CD95L, TRAIL/Apo-2L,

RANKL/OPGL/TRANCE, BAFF/BLyS/TALL-1, Fas/CD95, TRAIL-R1/DR4, TRAIL-R2/DR5, TRAIL-R3/DCR1, TRAIL-R4/DCR2, TRAMP/DR3 and TROY/TAJ . For the official TNF superfamily (TNFSF) nomenclature and additional synonyms, consult http://www.gene.ucl.ac.uk/nomenclature/genefamily/tnftop.html.

hypohidrotic ectodermal dysplasia (HED, EDA/EDAR) and familial expansileosteolysis (FEO, RANK) [10]. It is likely that other links between TNF members and diseases will be uncovered in the future.

\section{Structural features of TNF family ligands}

The THD is a 150 amino acid long sequence containing a conserved framework of aromaticand hydrophobic residues (Fig. 2). Todate, atomic-level structures are availablefor the THD of TNF $[20,21]$, LT $\alpha$ [22], CD40L [23] and TRAIL [24-27]. THDs sharea virtually identical tertiary fold and associate to form trimeric proteins (F ig. 3a). The THDs are $\beta$-sandwich structures containing two stacked $\beta$-pleated sheets each formed by fiveanti-parallel $\beta$ strands that adopt a dassical 'jelly-roll'topol ogy. Theinner sheet (strands $A, A^{\prime}, H, C$ and $F$ ) is involved in trimer contacts, and the outer sheet (strands B, $B^{\prime}$, $D, E$ and $G$ ) is exposed at thesurface. Trimeric THDs are $-60 \AA$ in height and resemblebell-shaped, truncated pyramids with variable loops protruding out of a compact core of conserved anti-parallel $\beta$ strands (Figs 2,3a). TRAIL is unique with respect to the $A A^{\prime}$ loop, which contains a 15 residue-long insertion that spans the whol eouter surface of the monomer $[24,26,27]$. The trimer is assembled such that oneedge of each subunit (strands $E$ and $F$ ) is packed against the inner sheet of its neighbour, forming large and mostly hydrophobic interfaces resulting in a very stableinteraction [20,26,27]. TNF and $C D 40 \mathrm{~L}$ contain a single disulfide bridgelinking the CD and EF loops [20,23] (Fig. 3a). Similar disulfide links are predicted to occur in F asL, LIGHT, 


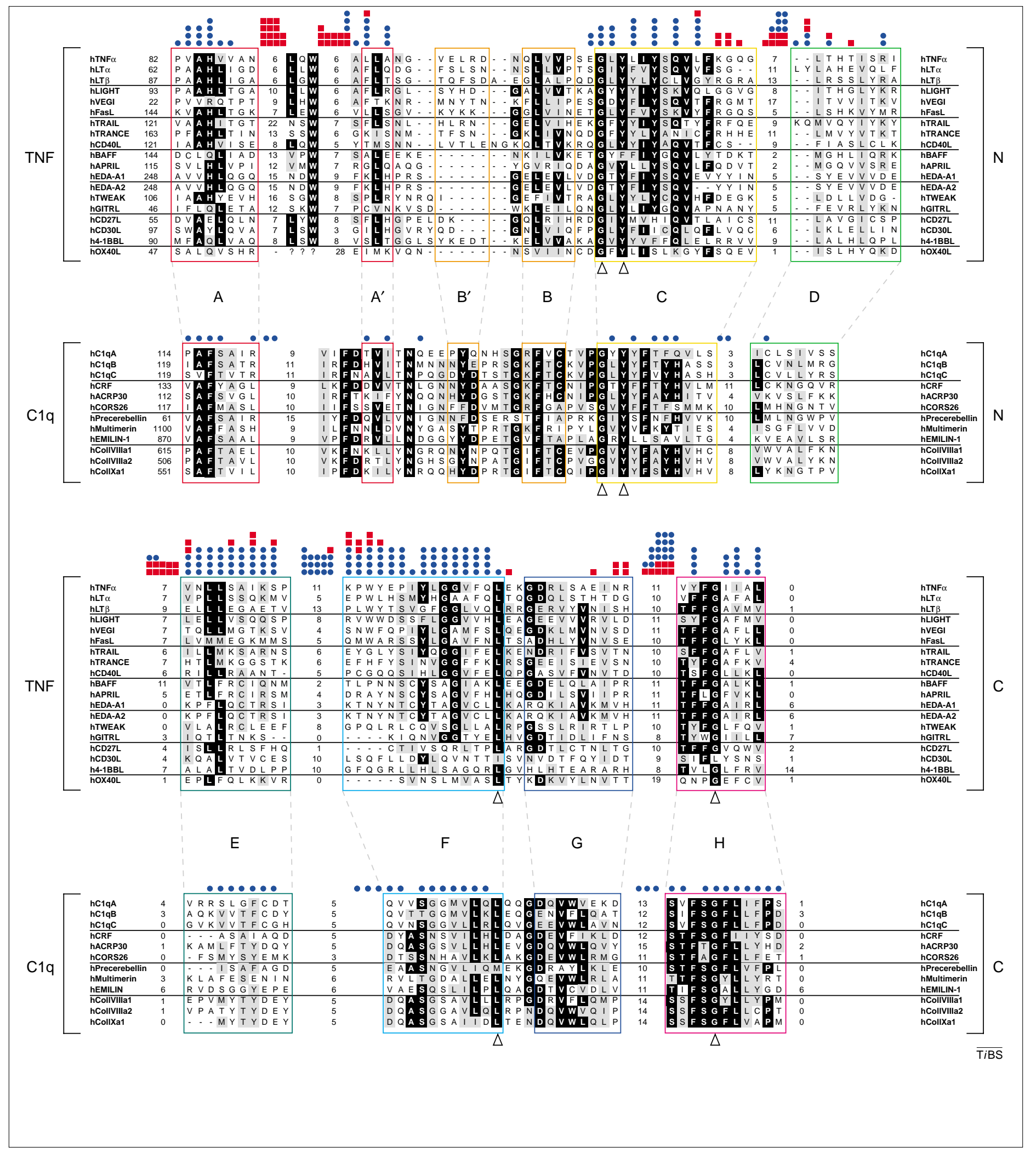

Fig. 2. Sequence alignment of the human tumour necrosis factor (TNF) and $\mathrm{Clq}$ superfamilies. Primary sequence alignments of the TNF homology domains (THDs) of 19 TNF ligands (including the distantly related member OX 40L) and of 12 published $\mathrm{C} 1 \mathrm{q}$-related proteins. The alignment has been reduced to regions of significant sequence homology. Intervening loops have been omitted except for the conserved $\mathrm{L} N \times \mathrm{W}$ motif in the $A A^{\prime}$ loop of the TNF ligands, but their length is indicated. The individual $\beta$ strands $(A-H)$ are highlighted with boxes coloured with respect to their succession in the primary structure from red to violet. Blue dots above the alignment indicate residues involved in monomer-monomer interface formation and their numbers represent the frequency at which each position is found to interact in the five structures available (e.g. TNF, LT $\alpha$, CD40L, TRAIL and mACRP30). Red squares represent residues involved in receptor binding in the two complex structures available (LT $\alpha$-TNF-R1 and TRAIL-DR5). Arrowheads underneath the sequences point to the four conserved residues in the TNF and Clq families. The multiple sequence alignment was generated with the amino acid sequence of THD and gClq domains using ClustalW, and was edited manually to account for structural knowledge. Identical amino acids (inverse) and 50\% similar amino acids (grey) were shaded using Boxshade. 
(a) $\mathrm{TNF} \alpha$

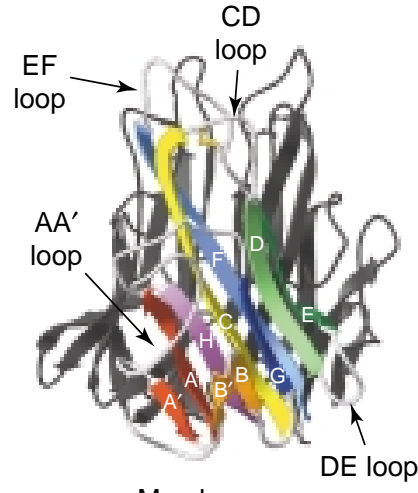

Membrane

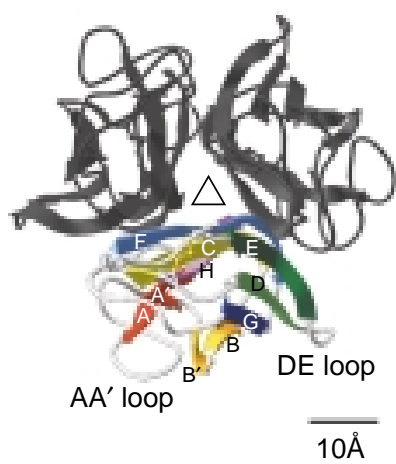

(b) ACRP30

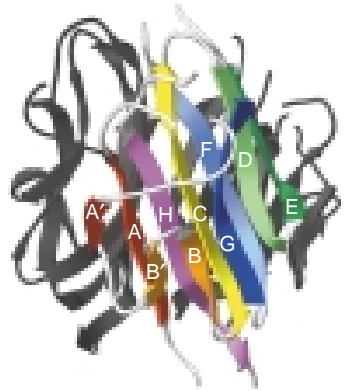

Collagen domain

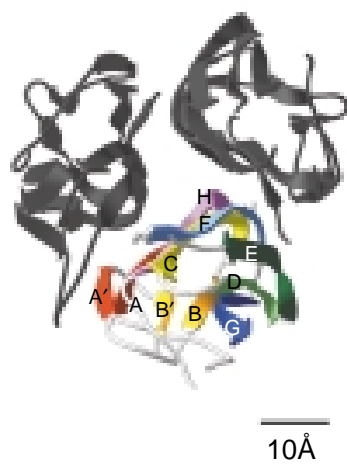

(c) Poliovirus

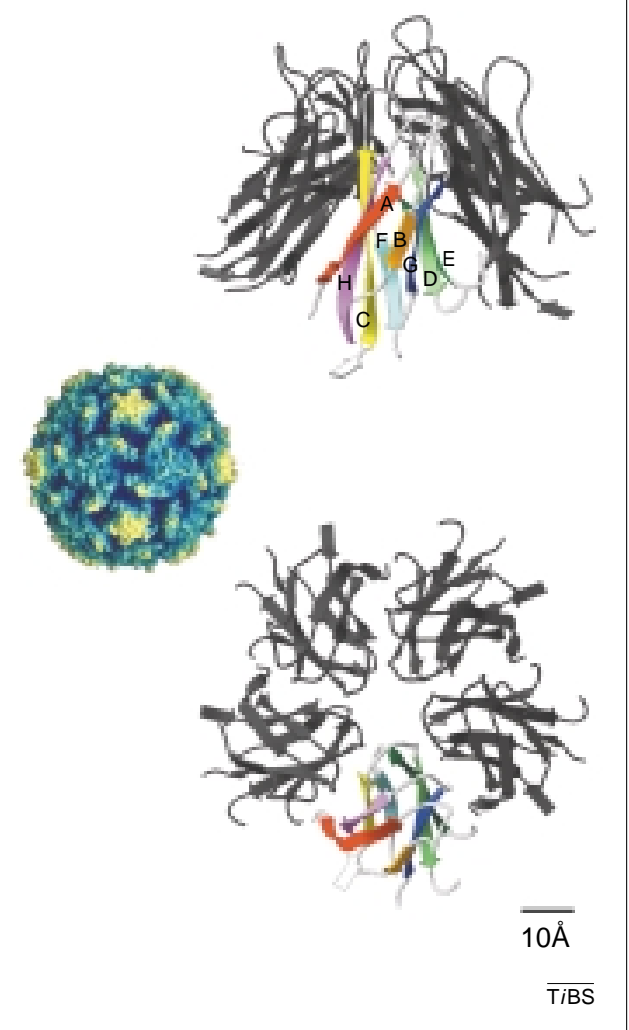

Fig. 3. The tumour necrosis factor (TNF) homology domain (THD). (a) Ribbon diagrams of the THD of human TNF seen from the side (left) and top (right) orientation. One monomer is highlighted and the other two are shaded. The ten anti-parallel $\beta$ strands (designated A, A', B', B, C, D, $\mathrm{E}, \mathrm{F}, \mathrm{G}$ and $\mathrm{H}$ according to Ref. [20]) are coloured using the same code as in Fig. 2. Intervening loops are shown in white. The orientation of the THD relative to the membrane is indicated. Note the close proximity of $\mathrm{N}$ and $\mathrm{C}$ termini. Models are based on the PDB atomic coordinate file 1TNF [20]. (b) Structure of the gClq domain of murine ACRP30 seen from the side (left) and top (right) orientation showing its similarity to the THD. Models are based on the PDB atomic coordinate file 1C28 [31]. (c) Representation of the pentameric 'jelly-roll' domain of the VP1 capsid protein (boxed in the representation of the viral particle in the centre) of the Mahoney strain of type 1 human poliovirus (PV) seen from the side (left) and top (right) orientation. Strands are coloured and numbered as in panels $A$ and $B$. The topological organization of the eight strands is identical to that of the THD, with the exception of the two interruptions in strands $A$ and $B$. Models are based on the PDB atomic coordinate file 2PLV [36]

VEGI, CD30L and CD27L, whereas TWEAK, EDA, APRIL and BAFF havea predicted disulfide bridge between $\beta$ strands $E$ and $F$. In TRAIL, a single cysteineresidue (Cys230) in theEF loop is involved in the coordination of a $\mathrm{Zn}(\mathrm{II})$ ion, with each monomer contributing to one coordination position whereas the fourth coordination position is occupied by an internal solvent moleculeor a chl oridecounter-ion [24-26]. This metal-binding site is unique so far in theTNF family and affects the stability and bioactivity of TRAIL $[26,28,29]$. I ncompleteZn coordination, and formation of partially oxidized, disulfide-linked species of TRAIL, has recently been suggested to account for its hepatotoxicity [30].

\section{TNF related structures - the Clq family}

Crystallographicstudies reveal ed that TNF and the gl obular gClq domain of mouseACRP 30 havea closely related tertiary structure and trimeric organization, suggestive of an evolutionary link between theTNF and Clq families [31] (Fig. 3a,b). Thehuman Clq genefamily comprises, so far, 13 members (Fig. 2), which are characterized by the presence of a trimeric gl obular C-terminal domain, known as gC 1q. The prototypical member of the family is Clq, a bouquet-like mol ecul ecomprising 18 chains (six each of $\mathrm{ClqA}, \mathrm{ClqB}$ and $\mathrm{ClqC}$ ) that associateinto six heter otrimeric $\mathrm{gC}$ lq domains held together by a bundle of collagen domains. Clq recognizes immune complexes and triggers the classical complement pathway (recently reviewed in Ref. [32]). The Clq family al so contains several coll lagenous members (CRF, ACRP 30, CORS26, EMILIN- 1 and -2 , and collagens VII and X) and two non-collagenous members (Precer ebellin and Multimerin) (Fig. 2). Many of these proteins are components of the extracellular matrix in diverse organs [32]. ACRP 30 is an abundant serum protein that is synthesized by adi posetissues in responseto insulin and is downregulated in obesemouseand humans $[33,34]$. The homologues of ACRP30 are drastically downregulated in theserum of hibernating Siberian chipmunks, pointing to a role in energy metabol ism. I ndeed, ACRP 30 induces weight loss in mice via activation of fatty acid catabolism 
[35]. Conserved residues of gClq domains are located within the core $\beta$ strands, as previously discussed for THD domains. Although the sequenceidentity between the two families is reduced to only four aminoacid residues (indicated with arrowheads in Fig. 2), the overall hydrophobic character of the internal $\beta$-pleated sheet is maintained in both families.

To date, there is no evidencethat the mode of interaction described in theTNF family al so applies to proteins containing gl obular Clq domains. Several Clq receptors have been described, but none of them, with thenotable exception of theimmunecomplex, binds to the globular domain. Nevertheless, the recent demonstration that thegClq domain of ACRP30 is biol ogically activeimplies the existence of ACRP30 receptor(s), which could be structurally related to TNF receptors.

\section{TNF related structures - viral capsid proteins}

As first noticed by J ones and E ck in 1989, the overall fold and topol ogy of the THD is very similar to that of the capsid proteins of small spherical plant viruses

(e.g. Tomato Bushy Stunt Virus and SatelliteTobacco Necrosis Virus) and mammalian picornaviruses (including the common human Rhinoviruses, the Foot-and-Mouth Disease Virus and Pol iovirus) despite no detectable similarity at the primary sequencelevel $[20,21]$. Although thesecapsid proteins associateal ong a fivefold instead of a threefol d axis of symmetry, the connectivity of their $\beta$ strands is identical to that of theTHD, with the exception that strands $A$ and $B$ arenot interrupted by loops [36] (Fig. 3c). These fivefold structures appear on the virus surface as 12 broad, star-like protuberances (Fig. 3c). Although the structural relationship existing between these apparently unrel ated protein families highlights the propensity of 'jelly-roll'motifs to ol igomerize, there appears to be no functional conservation between TNF family members and icosahedral viral capsid proteins. Indeed, the receptors all lowing entry of this class of viruses into cells do not bel ong to the TNF receptor family and do not bind directly to the ol igomeric 'jelly-roll'structure[37].

\section{The TNF receptor family}

In humans, 29TNF receptors haveso far been identified (Fig. 1). These are primarily type I (extracellular $\mathrm{N}$ terminus, intracellular $\mathrm{C}$ terminus) transmembrane proteins, but there are exceptions to this rule: BCMA, TACI, BAFFR and XEDAR aretype III transmembrane proteins (lacking a signal peptide), TRAI L-R3 is anchored by a coval entlylinked C-terminal glycolipid, and OPG and DcR3 lack a membrane-interacting domain and aretherefore secreted as soluble proteins. Sol uble receptors can al so begenerated by proteolytic processing (CD27, CD30, CD40, TNF-R1 and TNF-R2) [38], or by alternativesplicing of the exon encoding the transmembrane domain (F as and 4-1BB) [39]. The essential role of these soluble receptors in modulating the activity of their cognateligands has been welldocumented (for OPG and TNF-R1 examples, see Refs $[40,41])$. In addition, several viral open reading frames encode receptor homologues that interact with TNF and that are believed to interfere with the onset of inflammatory responses (SVF -T2 in Shopefibroma virus, Va53R in Vaccinia, cytokineresponse modifiers $\mathrm{CrmB}, \mathrm{CrmC}$ and $\mathrm{CrmD}$ in orthopoxviruses) (reviewed in Ref. [39]). The TNF receptor family member NGFR is unique in that it binds low-affinity ligands that do not belong to the TNF family. Theseligands (NGF, BDNF and neurotrophins) al so engage a family of high-affinity tyrosinereceptor kinases (trkA, B and C), which are unrel ated to TNF receptors [42]. The existence of a bona fideTNF ligand for NGF R cannot be excluded at present.

\section{Structural features of the TNF receptor family}

The extracellular domains of TNF receptors are characterized by the presence of cysteine-rich domains (CRDs), which are pseudo-repeats typically containing six cysteine residues engaged in the formation of three disulfide bonds. The number of CRDs in a given receptor varies from one to four, except in the case of CD30 where the three CRDs have been partially duplicated in the human but not in the mouse sequence. The repeated and regular arrangement of CRDs confers an el ongated shape upon the receptors, which is stabilized by a slightly twisted ladder of disulfide bridges ( $F$ igs 1,4 ). Sequence alignment of TNF receptor family members in the absence of structural information is difficult because the spacing of cysteine residues is not al ways conserved between receptors. Naismith and Sprang have introduced a classification based on distinct structural modules that greatly facilitates sequence comparison between TNF receptors [43] (Fig. 5). Each module type is designated by a letter (A, B, C and N for crystallized modules, and $X$ for modules of unknown structure) and by a numeral indicating the number of disulfide bridges it contains. A typical CRD is usually composed of an A1-B2 or A2-B1 module or, less frequently, a different pair of modules. A1 modules are 12-27 amino acids long, consist of three short $\beta$ strands linked by turns, and contain a single disulfide bridge connecting strands 1 and 3 , yielding a characteristic C-shaped structure (Fig. 4). A2 modules contain a second disulfide bridge linking the second and third strands without affecting the overall structure. B modules are 21-24 amino acids long and comprise three anti-parallel strands adopting an S-shaped fold reminiscent of a paper clip (Fig. 4). In this case, the fold is constrained by two entangl ed disulfide bridges linking strands 1 and 3 in B2 modules. The first disulfide bridge is replaced by a hydrogen bond in B1 modules [43]. The 


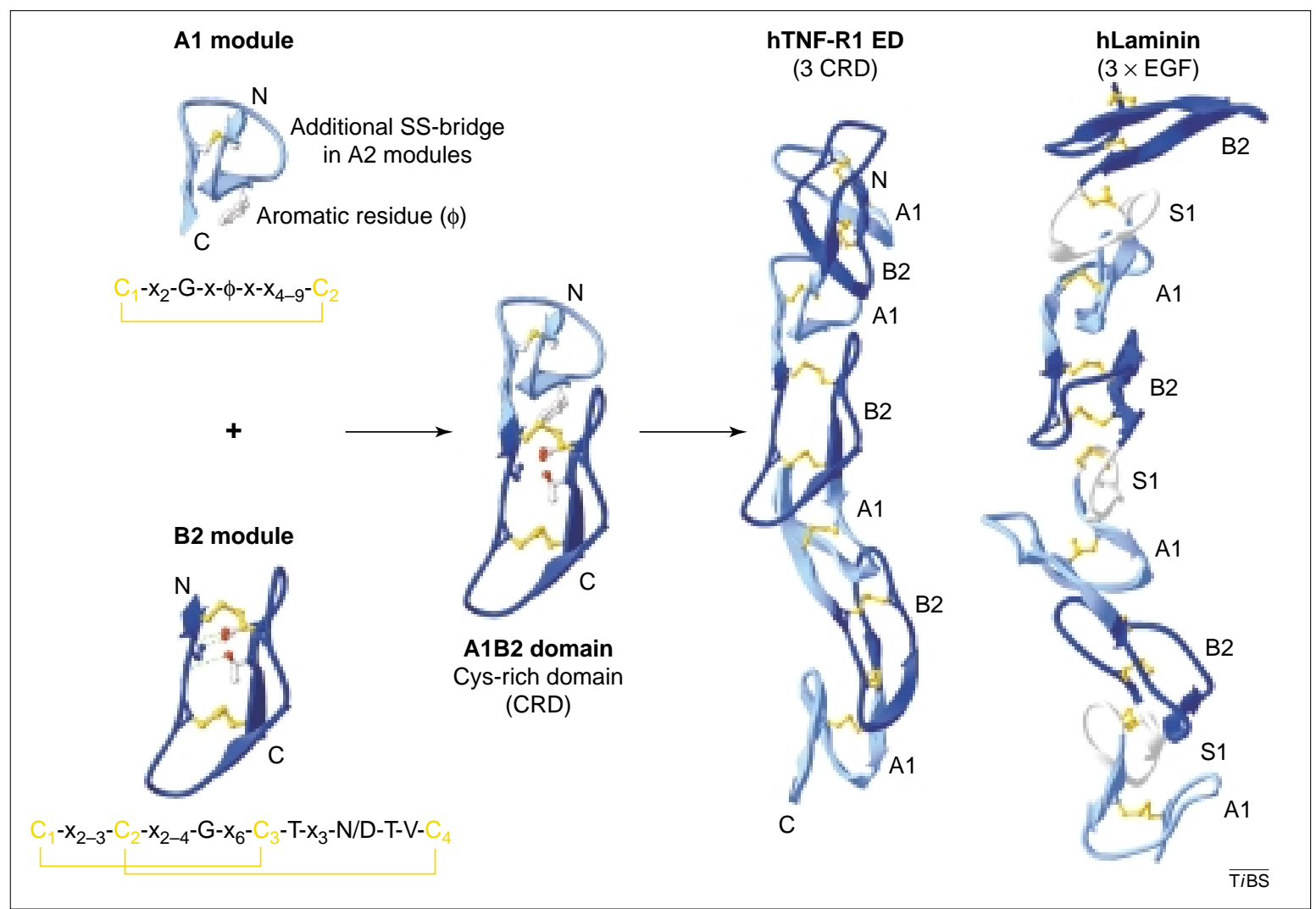

Fig. 4. Modular organization of the extracellular domain of the tumour necrosis factor (TNF) receptors. Ribbon representation of an A1 module (light blue, top left) and a B2 module (dark blue, bottom left) along with their consensus sequence. In the middle, the two modules are combined to generate a cysteine rich domain (CRD). The full extracellular domain contains several CRDs stacked on top of each other. The tertiary structure of the epidermal growth factor (EGF) domains of human laminin $\gamma 1$ chain is remarkably similar, except that an additional loop is inserted between CRDs ( $S 1$, shown in white). Models are based on the PDB atomic coordinate files 1TNR (TNF receptor) [22] and 1KLO (laminin $\gamma 1$ ) [48].

structure of $A$ and $B$ modules is al so reflected at the level of the primary sequence by the conservation of a few non-cysteineresidues (see consensus sequence in Fig. 4). Other modules are less frequent. So far, the $\mathrm{N}$-terminal $\mathrm{N} 1$ modules have been found only in the TRAIL receptors, in which they precede the first A1-B2 CRD. Structurally, the $N 1$ module resembles the second half of a $B$ module $[24,25,27]$. The fourth CRD of TNF-R1 contains an A1-C2 module pair, in which the cysteine connectivity of $C 2$ is distinct from that of a B2 module. TACI , BCM A and $\mathrm{Fn} 14$ also contain putative $\mathrm{A} 1-\mathrm{C} 2 \mathrm{CRDs}$, but these remain to be demonstrated at the structural level. Finally, we have collectively designated, as X2, four unrelated modules of unknown structure that are found in TRAMP, GITR, BAFFR and viral CrmC. The recently described BAF $F$ receptor (BAFFR) [44] contains a single $X 2$ module whose sequence resembles an $A$ module entangled with the beginning of a $B$ module (Fig. 5). Morestructural work is needed to understand the molecular interaction of this receptor with BAFF. TNF receptors are often viewed as monomers, principally

because they appear in this form in crystal structures of ligand-receptor complexes. However, TNF-R 1 has al so been crystallized as both head-tohead and head-to-tail dimers [45], and there is genetic and experimental evidence that Fas, TNF $\mathrm{R} 1$ and $\mathrm{CD} 40$ exist as preformed oligomers within the plasma membrane [46]. Self-association of the receptors depends on an $\mathrm{N}$-terminal pre-ligand association domain (PLAD) that includes the first CRD and that is not directly involved in ligand binding.

\section{TNFR-related structures - the EGF-like domain}

$A 1$ and $B 2$ modules are not restricted to the TNF receptor family but also form the structural basis of epidermal growth factor (EGF)-like domains present in several proteins such as laminins. Laminins are composed of threerelated chains ( $\alpha, \beta$ and $\gamma$, of which therearedifferent isoforms) associated by a C-terminal coiled-coil domain. Thesechains display several gl obular domains in their $\mathrm{N}$-terminal moieties with intervening, el ongated structures composed of EGF-likerepeats [47]. As shown in Fig. 4, theoverall structure of EGF-like and CRD repeats is strikingly similar, except that A1-B2 modules in laminin are separated by an additional module, which wehave designated S1 because of its small size [48]. EGF-like repeats 3-5 of the $\gamma 1$ chain of laminin bind Nidogen- 1 , a protein that interconnects laminin mol ecules in the basement membrane, but whose sequence is unrelated toTNF or Clq family members. Sofar, thereis no evidence that EGF-like repeats bind TNFor Clq-likeligands. 
Fig. 5. Sequence alignment of tumour necrosis factor (TNF) receptor modules. (a) Sequence alignment of TNF receptor modules in their order of appearance from the $\mathrm{N}$ to the

$\mathrm{C}$ terminus (left to right and top to bottom). Conserved cysteine residues are boxed whereas similar residues are shaded. The connectivity of the cysteines is shown at the top. A star marks modules that do not entirely obey canonical cysteine patterns of $A, B, C$ and $\mathrm{N}$ modules. Receptors containing $\mathrm{C} 2$ modules are shown at the bottom of the main alignment. A putative disulfide connectivity is indicated, based on the known structure of the $\mathrm{C} 2$ module of TNF-R1 (b) Sequence alignment of individual A1, B2 and intervening $\mathrm{S} 1$ modules of three epidermal growth factor (EGF) domains of human laminin $\gamma 1$ chain. (a) $A$ and $\mathbf{N}$ modules

B modules

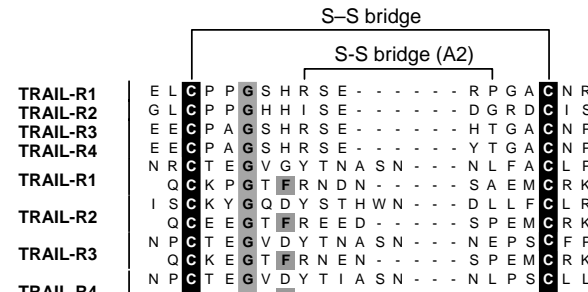

TRAIL-R4 SPEMIC

A P CPDHYYTDSWH

TS D EEL

RANK

TRAMP *

Fn14

DR

TNF-B2

TNF-R1

$K$ K C P D GFFSNETS
PPCT SEKHYE.

LPCG PDEYLDSWN
A C T A G Y LWS Q.

$K P C L A G Y F S D A F$
$P B C D C A G D F H K K$

$\begin{array}{ll}S K A P & \\ H L G R & \end{array}$

EEDKC L

$P R$
$L C D C A G D F H K K$
$L V C P D T F$

$G T C L P G F Y E$.
A PCS RGSS

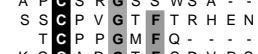

$K Q C A R G T F S D V P$

DSCEDSTYTQLWN

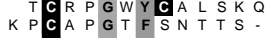

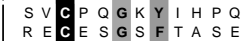

LT $\beta-\mathbf{R}$

GCRKNQY

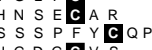

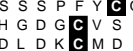

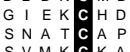

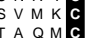

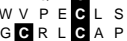

G C R
$S T$ D

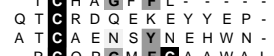

NNS

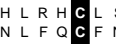

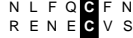

(1)

HVEM

A
$V P C K A A G H F Q N T S S$

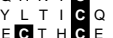

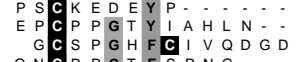

SA

DcR3

QN CPPGTFSPNG.

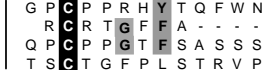

GLLSK

Fas

CD40

$\mathrm{CD}$

CD30

CD27

NGFP

NGFR

4-1BB

ox40

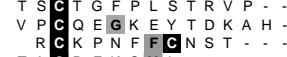

TACREK Q Y L

LPCGESEFLDTWN
EP P P V G F F S T Y S S

D
DT CHGNPSHYYDK

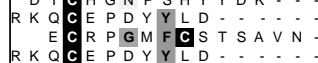

K K QCEPDYYLD

K $S$ C R P E G M I GA T S A T N

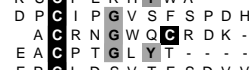

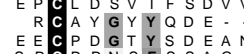

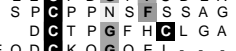

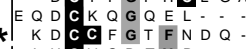

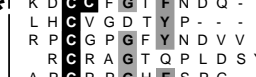

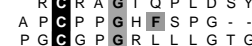

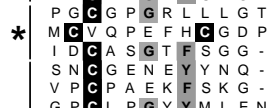

EDAR

XEDAR

YSC

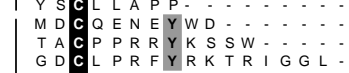

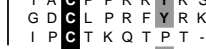

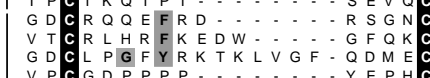

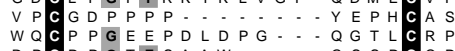

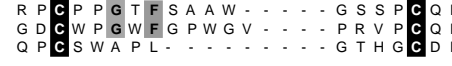

RELT

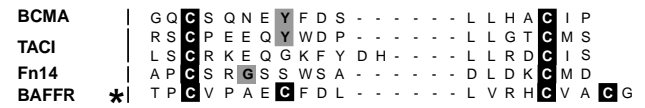

(b)

\section{A modules}

Lamini

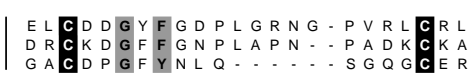

TNF-R1

LT $\beta-R$
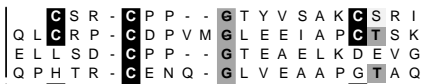

HVEM

DcR3

Fas

CD40

CD30

CD27

NGFR

4-1BB

ox40

GITR

EDAR

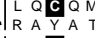

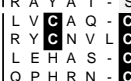

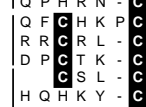

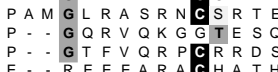

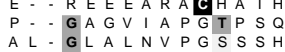

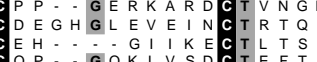

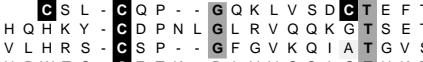

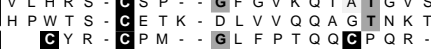

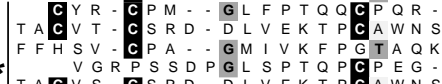

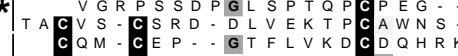

ES R R R :

KPCTE CVG LOSMSAPCVEA

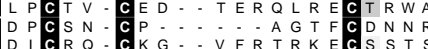

RPWTN-GSLD-GKSVLVNGTKER

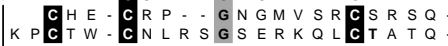

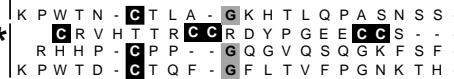

TNF-R1

\section{B modules}

Lamini

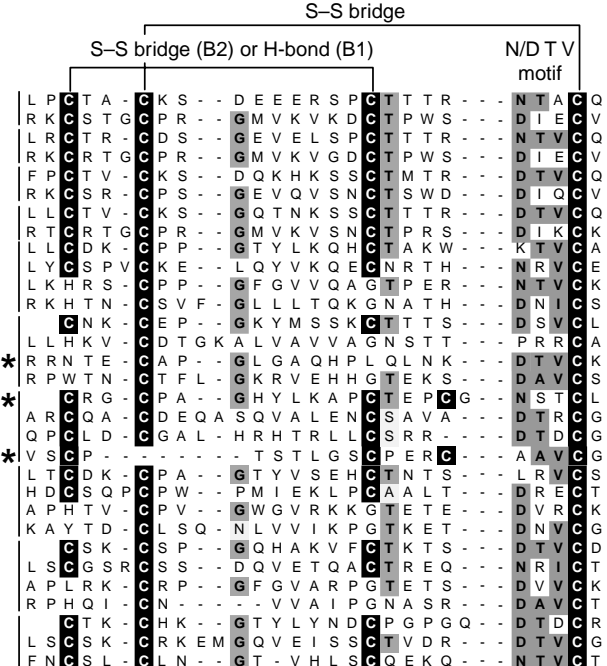

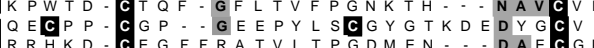

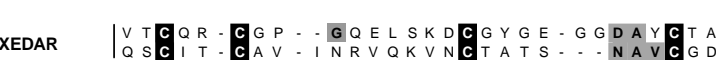

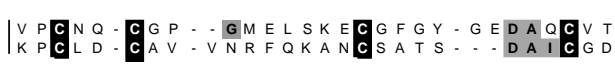

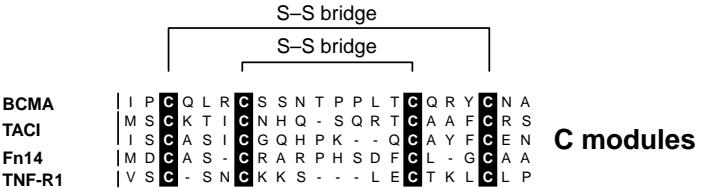

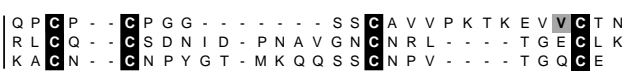




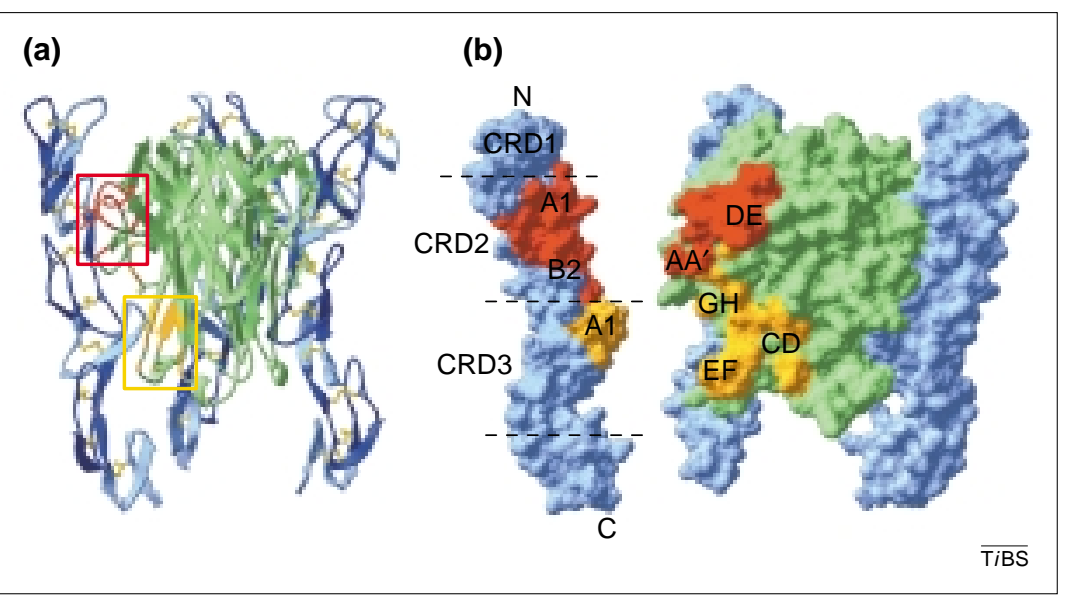

Fig. 6. Receptor-ligand interactions. Ribbon and space-filling representations of the lymphotoxin $\alpha$ (LT $\alpha$ )-TNF-R1 3:3 complex. LT $\alpha$ is shown in green, TNF-R1 in blue, and the two interaction surfaces in red and orange, respectively. In panel (a), the side chain of Tyr 142 is shown. (b) is an open-book representation revealing the interaction regions. Loops, modules and cysteine-rich domains (CRDs) contributing to these interaction surfaces are identified. Models are based on the PDB atomic coordinate files 1TNR [22]

\section{Acknowledgements}

We thank Kimberly Burns for critical reading of the manuscript. The molecular models shown in this publication were generated using Swiss-PDB Viewer v3.7 $\beta 2$ [52], which can be freely downloaded from http://www.expasy.ch/spd bv/. This work was supported by grants from the Swiss National Science Foundation to P.S. and J.T. In 1993, Banner and colleagues [22] published a seminal study unraveling the first structure of a TNF ligand $(L T \alpha)$ bound to its cognate receptor (TNF-R1). Theasymmetric unit contains three receptors and three ligands assembled as a hexameric complex in which a singleTNF trimer binds to three receptor molecules (F ig. 6). More recently, highly similar crystal structures have been reported for complexes between TRAIL and TRAI L$\mathrm{R} 2$, confirming that the $3: 3$ stoichiometry is the likely basis of the signaling unit [24,25,27]. Indeed, the geometry of the receptor-ligand complex matches that of TRAF -2, a trimeric intracellular adaptor molecule mediating TNF-R2 and CD40 signals [49]. The recently identified receptors $B C M A, T A C l, B A F F R$ and $\mathrm{Fn} 14$ do not contain the A1-B2-A1 succession of modules involved in the binding of both TNFR 1 and TRAILR2 to their respectiveligands, implying that distinct receptor-ligand interfaces must exist. However, the threefold geometry is alsolikely to be conserved for theseligand-receptor complexes.

The receptor molecules bind with their long axis roughly parallel to the $\mathrm{C}_{3}$ symmetry-axis of the trimeric ligand, in the groove formed by the interface of each pair of monomers. Conformational changes occurring upon complex formation are relatively minor, and only substantially affect receptor-contacting loops of the ligand ( $C D$ and $A^{\prime} A^{\prime}$ ) $[22,25]$. There are mainly two contact regions between the receptor and the ligand. The first contact area involves receptor residues corresponding to the second CRD of the receptor (A1 plus half of B2) and loops DE and $A^{\prime} A^{\prime}$ of two adjacent ligand subunits (Fig. 6, region shown in red). This area is based on a central hydrophobic interaction containing a relatively conserved tyrosine residue (present in loop DE of TNF, LT $\alpha$,
Interactions between a TNF ligand and a TNF receptor
FasL, TRAIL, LIGHT and VEGI) that is crucial for receptor binding in TNF, LT $\alpha, F$ asL and TRAIL. In the second, more polar interaction region, the remainder of the second CRD (second half of $B 2$ ), and the 11 module of the third CRD, of TNF-R 1 make contacts with the $C D$ and $E F$ loops of two adjacent ligand subunits (F ig. 6, region shown in orange). In addition, Cha and colleagues have provided evidence for the existence of a third, central interaction region in their TRAIL-TRAILR2 structure. The central region involves residues 131-135 of theAA'loop that penetrates into the central interaction region upon binding, forming several specific polar interactions. This additional interaction patch might well be specific to TRAIL because of its long AA' loop [24].

As expected, regions of contact between ligands and receptors are very diverse among family members and contribute to the specific interaction of ligand-receptor pairs (Fig. 2). However, prediction of receptor-ligand interactions is not straightforward as different ligands can bind the same receptor (e.g. both TNF and $L T \alpha$ bind TNF-R 1 ) whereas almost identical ligands can bind different receptors. In this context, the particular case of ectodysplasin A (EDA) is interesting as two of its isoforms (namely EDA-A1 and -A2), which differ by only two aminoacids, display a mutually exclusive receptor specificity (for EDAR and XEDAR, respectively). In this case, removal of the residues Glu308-Val 309 is predicted to suppress a negative chargein the second receptor interaction site of EDA [50].

Thereceptor Herpes virus entry mediator (HVEM) interacts with twoligands of the TNF family (LIGHT and $L T \alpha$ ), but is also hijacked by the viral glycoprotein $D(g D)$ of herpes simplex virus. The latter interaction invol ves the first B2 module of HVEM and is structurally unrelated tothat of $a$ regular TNF ligand with a TNF receptor [51].

\section{Conclusions}

The past few years have witnessed a dramatic increase in thenumber of theTNF and TNF receptor family members. This was a direct consequence of expressed sequencetag sequencing projects combined with the devel opment of bioinformatic tools. With the completion of the genome sequencing project, it is now reasonable to assume that these two families approach their definitivesizes. They are characterized by a conserved molecular architecture and mode of interaction. A few more unexpected additions might arise from expression cloning of receptors, becausetheir modular structureis more diversethan that of theligands. Conversely, novel ligand specificities might arisefrom alternative splice variants or from the heteromericassociation of known ligands. The molecular characterizations of TNF and TNFR have provided a basis for the understanding of their biological roles, and their implication in genetic diseases. 


\section{References}

1 Black, R.A. et al. (1997)A metalloproteinase disintegrin that releases tumour-necrosis factor- $\alpha$ from cells. Nature 385, 729-733

2 Lum, L. et al . (1999) Evidencefor a role of a tumor necrosis factor- $\alpha$ (TNF- $\alpha$ )-converting enzyme-like protease in shedding of TRANCE, a TNF family member involved in osteoclastogenesis and dendritic cell survival. J . Biol. Chem. 274, 13613-13618

3 Powell, W.C. et al. (1999) Themetalloproteinase matrilysin proteolytically generates activesoluble Fas ligand and potentiates epithelial cell apoptosis. Curr. Biol. 9, 1441-1447

4 Chen, Y. et al. (2001) Mutations within a furin consensus sequence bl ock proteolytic release of ectodysplasin-A and causeX-linked hypohidrotic ectodermal dysplasia. Proc. Natl. Acad. Sci. U.S. A. $98,7218-7223$

5 Schneider, P. et al. (1999) BAFF, a novel ligand of thetumor necrosis factor family, stimulates B cell growth.J . Exp. Med. 189, 1747-1756

6 Schneider, P. etal. (2001) Mutations leading to Xlinked hypohidrotic ectodermal dysplasia affect threemajor functional domains in thetumor necrosis factor family member ectodysplasin-A. J . Biol. Chem. 276, 18819-18827

7 Tanaka, M. et al. (1998) Downregulation of Fas ligand by shedding. Nat. Med. 4, 31-36

8 Browning, J .L. etal. (1995) Characterization of surfacelymphotoxin forms. Use of specific monocl onal antibodies and sol uble receptors. J . Immunol. 154, 33-46

9 Mariani, S.M. and Krammer, P.H. (1998) Differential regulation of TRAIL and CD95 ligand in transformed cells of the T and B lymphocyte lineage. Eur.J . I mmunol. 28, 973-982

10 Locksley, R.M. etal. (2001) TheTNF and TNF receptor superfamilies: integrating mammalian biology. Cell 104, 487-501

11 Fu, Y.X. and Chaplin, D.D. (1999) Development and maturation of secondary lymphoid tissues. Annu. Rev. I mmunol. 17, 399-433

12 Vogel, L.A. and Noelle, R.J . (1998) CD40 and its crucial roleas a member of theTNFR family. Semin. I mmunol. 10, 435-442

13 Mackay, F. etal. (1999) Micetransgenic for BAFF devel op lymphocytic disorders al ong with autoimmunemanifestations. J . Exp. Med. 190, 1697-1710

14 Gross, J .A. et al. (2000) TACl and BCMA are receptors for a TNF homol ogue implicated in Bcell autoimmune disease. Nature404, 995-999

15 Gravestein, L.A. and Borst, J. (1998) Tumor necrosis factor receptor family members in the immunesystem. Semin. Immunol. 10, 423-434

16 Anderson, D.M. et al. (1997) A homologue of the TNF receptor and its ligand enhance T-cell growth and dendritic-cell function. Nature 390, 175-179

17 Bennett, S.R. etal. (1998) Hel p for cytotoxic-T-cell responses is mediated by CD40 signalling. Nature 393, 478-480

18 Krammer, P.H. (2000) CD95's deadly mission in theimmunesystem. Nature407, 789-795

19 Dinarello, C.A. (2000) Proinflammatory cytokines. Chest 118, 503-508

20 Eck, M.J . and Sprang, S.R. (1989) Thestructure of tumor necrosis factor-al pha at $2.6 \AA \AA$ resolution. Implications for receptor binding. J . Biol. Chem. 264, 17595-17605

$21 \mathrm{~J}$ ones, E.Y. et al. (1989) Structure of tumour necrosis factor. Nature 338, 225-228

22 Banner, D.W. et al. (1993) Crystal structure of the solublehuman $55 \mathrm{kdTNF}$ receptor-human TNF $\beta$ complex: implications for TNF receptor activation. Cell 73, 431-445

23 Karpusas, M. et al. (1995) $2 \AA$ crystal structure of an extracellular fragment of human CD40 ligand. Structure3, 1031-1039

24 Cha, S.S. et al . (2000) Crystal structure of TRAIL-DR5 complex identifies a critical role of the unique frameinsertion in conferring recognition specificity. J . Biol. Chem. 275, 31171-31177

25 Hymowitz, S.G. et al. (1999) Triggering cell death: the crystal structure of Apo2L/TRAIL in a complex with death receptor 5 . Mol. Cell 4, 563-571

26 Hymowitz, S.G. et al. (2000) A uniquezincbinding sitereveal ed by a high-resolution X-ray structure of homotrimericApo2L/TRAIL. Biochemistry 39, 633-640

27 Mongkolsapaya, J .et al. (1999) Structure of the TRAIL-DR5 complex reveals mechanisms conferring specificity in apoptotic initiation. $N$ at. Struct. Biol. 6, 1048-1053

28 Bodmer, J .L. et al. (2000) Cysteine 230 is essential for the structureand activity of the cytotoxic ligand TRAIL.J . Biol. Chem. 275, 20632-20637

29 Trabzuni, D. etal. (2000) Functional analysis of tumour necrosis factor- $\alpha$-related apoptosisinducing ligand (TRAIL): cysteine-230 plays a critical rolein the homotrimerization and biological activity of this novel tumoricidal cytokine. Biochem. J . 350, 505-510

30 Lawrence, D. et al. (2001) Differential hepatocyte toxicity of recombinant A po2L/TRAIL versions. Nat. Med. 7, 383-385

31 Shapiro, L. and Scherer, P.E. (1998) The crystal structure of a complement-1q family protein suggests an evolutionary link totumor necrosis factor. Curr. Biol. 8, 335-338

32 Kishore, U. and Reid, K.B. (2000) C1q: structure function, and receptors. I mmunopharmacol ogy 49, $159-170$

$33 \mathrm{Hu}$, E. et al. (1996) AdipoQ is a novel adipose specific genedysregulated in obesity. J. Biol. Chem. 271, 10697-10703

34 Scherer, P.E. et al. (1995) A novel serum protein similar to Clq, produced exclusively in adipocytes. J . Biol. Chem. 270, 26746-26749

35 Fruebis, J et al. (2001) Proteolytic cleavage product of 30-kDa adipocyte complement-related protein increases fatty acid oxidation in muscle and causes weight loss in mice. Proc. Natl. Acad. Sci. U. S. A. 98, 2005-2010

36 Hogle, J.M. et al. (1985) Threedimensional structure of poliovirus at $2.9 \AA$ resolution. Science 229, 1358-1365

37 Rossmann, M.G. etal. (2000) Cell recognition and entry by rhino- and enteroviruses. Virol ogy 269 , 239-247

38 Gruss, H.J . and Dower, S.K. (1995) Tumor necrosis factor ligand superfamily: invol vement in the pathol ogy of malignant lymphomas. Blood 85 , 3378-3404

39 Smith, C.A. et al. (1994) TheTNF receptor superfamily of cellular and viral proteins: activation, costimulation, and death. Cell 76, 959-962

40 McDermott, M.F. etal. (1999) Germlinemutations in the extracellular domains of the $55 \mathrm{kDaTNF}$ receptor, TNFR1, definea family of dominantly inherited autoinflammatory syndromes. Cell 97 133-144

41 Simonet, W.S. etal. (1997) Osteoprotegerin: a novel secreted protein involved in the regulation of bone density. Cell 89, 309-319

42 Yano, H. and Chao, M.V. (2000) Neurotrophin receptor structureand interactions. Pharm. Acta Helv. 74, 253-260

43 Naismith, J.H. and Sprang, S.R. (1998) Modularity in theTNF-receptor family. Trends Biochem. Sci. 23, 74-79

44 Thompson, J .S. et al. (2001) BAFF-R, a novel TNF receptor that specifically interacts with BAFF. Science293, 2108-2111

45 Naismith, J.H. etal. (1996) Structures of the extracellular domain of thetypel tumor necrosis factor receptor. Structure4, 1251-1262

46 Siegel, R.M. et al. (2000) F as preassociation required for apoptosis signaling and dominant inhibition by pathogenic mutations. Science288, 2354-2357

47 Tunggal, P. et al. (2000) Laminins: structureand geneticregulation. Microsc. Res. Tech. 51, 214-227

48 Stetefeld, J . et al. (1996) Crystal structure of three consecutivelaminin-typeepidermal growth factor-like (LE) modules of laminin $\gamma 1$ chain harboring thenidogen binding site.J. Mol. Biol. 257, 644-657

49 Park, Y.C. et al. (1999) Structural basis for selfassociation and receptor recognition of human TRAF 2. Nature 398, 533-538

50 Yan, M. et al. (2000) Two-aminoacid molecular switch in an epithelial morphogen that regulates binding to two distinct receptors. Science290, 523-527

51 Carfi, A . et al. (2001) Herpes simplex virus glycoprotein $D$ bound to the human receptor HveA. Mol. Cell 8, 169-179

52 Guex, N. et al. (1999) Protein modelling for all. Trends Biochem. Sci. 24, 364-367

53 Marsters, S.A. et al. (1998) I dentification of a ligand for the death-domain-containing receptor Apo3. Curr. Biol. 8, 525-528 